Tratamiento del aneurisma de aorta infrarrenal roto: cirugía abierta versus tratamiento endovascular

Treatment of ruptured abdominal aortic aneurysm: open surgical repair versus endovascular repair 


\section{OR 256}

Tratamiento del aneurisma de aorta infrarrenal roto: cirugía abierta versus tratamiento endovascular

Treatment of ruptured abdominal aortic aneurysm: open surgical repair versus endovascular repair

Recibido: 26/12/2020

Aceptado: 28/05/2021

Óscar Uclés Cabeza ${ }^{1}$, Isaac Martínez López¹, Ferrán Plá Sánchez², Adriana Baturone Blanco ${ }^{1}$, Francisco Javier Serrano Hernando ${ }^{1}$

${ }^{1}$ Servicio de Angiología, Cirugía Vascular y Endovascular. Hospital Clínico San Carlos. Madrid. ${ }^{2}$ Servicio de Angiología, Cirugía Vascular y Endovascular. Hospital Universitario de Gran Canaria Doctor Negrín. Las Palmas de Gran Canaria, Las Palmas

Correspondencia: Óscar Uclés Cabeza. Servicio de Angiología, Cirugía Vascular y Endovascular. Hospital Clínico San Carlos. C/ Profesor Martín Lagos, s/n. 28040 Madrid

e-mail: oscar.ucles@gmail.com

DOI: 10.20960/angiologia.00256

Financiación: la presente investigación no ha recibido ayudas específicas provenientes de agencias del sector público, sector comercial o entidades sin ánimo de lucro.

Conflicto de intereses: los autores declaran no tener ningún conflicto de interés.

\section{RESUMEN}


Introducción: el aneurisma de aorta abdominal roto (AAAr) es una patología que asocia una elevada morbimortalidad. El objetivo es analizar los resultados del tratamiento del AAAr en nuestro centro, comparando cirugía abierta (CA) y endovascular (EVAR).

Material y métodos: estudio de cohortes retrospectivo de pacientes intervenidos por AAAr con cuello infrarrenal entre enero de 2006 y diciembre de 2017, dividido en grupo CA y grupo EVAR. Análisis comparativo de comorbilidad, características anatómicas, técnica y resultados.

Resultados: 54 pacientes fueron incluidos, 26 (48,1\%) en el grupo CA y 28 $(51,9 \%)$ en el EVAR. Sin diferencias en cuanto a edad $(70,7 \pm 7,8$ años vs. 72,5 $\pm 9,5, p=0,45)$ ni comorbilidades, salvo la dislipemia $(26,9 \%$ vs. $67,9 \% p=$ $0,003)$. Los aneurismas eran mayores en el grupo CA $(88,1 \pm 17,9 \mathrm{~mm}$ vs. 72,4 $\pm 16 \mathrm{~mm}, \mathrm{p}=0,02$ ), con un mayor porcentaje de aneurisma iliaco asociado (34,6 \% vs. 17,8 \%, p = 0,07). Un 65,4 \% del grupo CA presentó inestabilidad hemodinámica preoperatoria, frente al $60,7 \%$ del EVAR $(p=0,72)$. Se empleó anestesia local en el $50 \%$ del grupo EVAR. Las necesidades transfusionales intraoperatorias medianas para el grupo CA y EVAR fueron $11 \pm 6$ y $4 \pm 3$ concentrados de hematíes respectivamente $(p=0,001)$. La mortalidad hospitalaria fue mayor en el grupo CA $(46,2 \% v s .28,6 \%)(p=0,18)$. En dicho grupo hubo 4 éxitus intraoperatorios. Un $54,5 \%$ de pacientes del grupo CA y un $64,3 \%$ del EVAR $(p=0,48)$ presentaron complicaciones mayores, siendo las más frecuentes la intubación prolongada y el fracaso renal que requirió terapia de reemplazo renal. La tasa de reintervención hospitalaria fue $18,2 \%$ en el grupo CA y $25 \%$ en el EVAR $(p=0,56)$, siendo en este grupo todas las reintervenciones en el subgrupo de endoprótesis aortouniiliaca. La supervivencia a 2 años fue del $51,7 \%$ en el grupo CA y del $65,2 \%$ en el EVAR $(p=0,28)$.

Conclusiones: el tratamiento del AAAr se asocia a una elevada morbimortalidad, y el EVAR puede mejorar los resultados, especialmente si se implanta una endoprótesis bifurcada.

Palabras clave: Aneurisma de aorta abdominal. Aneurisma roto. EVAR. Cirugía abierta. Mortalidad. 


\section{ABSTRACT}

Background: the ruptured abdominal aortic aneurysm (rAAA) is associated with high morbimortality. The purpose of this study was to compare results of open surgical repair (OSR) and EVAR in our institution in the management of rAAA.

Material and methods: retrospective observational cohort study was conducted on rAAA patients with infrarenal neck between January 2006 and December 2017. Sample was divided according to intervention: OSR vS EVAR. Comorbidities, anatomical features, intervention and results were analyzed by repair method.

Results: fifty-four patients were included, 26 (48.1\%) using OSR and 28 (51.9\%) using EVAR. Age (70.7 \pm 7.8 years vs. $72.5 \pm 9.5, p=0.45)$ and comorbidities showed no statistical significance, except for dyslipidemia (26.9\% vs $67.9 \% \mathrm{p}=0.003)$. Aneurysms were larger in OSR group (88.1 \pm $17.9 \mathrm{~mm}$ vs $72.4 \pm 16 \mathrm{~mm}, \mathrm{p}=0.02$ ), with a higher rate of associated iliac aneurysm ( $34.6 \%$ vs $17.8 \%, p=0.07$ ). $65.4 \%$ of patients in OSR group showed preoperative hemodynamic instability, compared to $60.7 \%$ in EVAR group $(p=0.72) .50 \%$ of EVAR procedures were performed under local anesthesia. Median intraoperative transfusion requirements for OSR group and EVAR group were, respectively, $11 \pm 6$ and $4 \pm 3$ red blood cell concentrates ( $p$ $=0.001)$. Hospital mortality was higher in OSR group $(46.2 \%$ vs $28.6 \%)$ ( $p=$ 0.18). There were four intraoperative deaths in this group. $54.5 \%$ of OSR patients and $64.3 \%$ of EVAR presented major complications. The most common ones were longtime intubation and acute renal failure requiring renal replacement therapy. Reintervention rate was $18.2 \%$ in CA group and $25 \%$ in EVAR group $(p=0.56)$, all reinterventions in EVAR group performed in rAAAs repaired by an aortouniiliac device. Two-year survival rate was $51.7 \%$ in CA group and $65.2 \%$ in EVAR group ( $p=0.28$ ).

Conclusions: the treatment of rAAA still associates with high morbimortality rates. However, EVAR could improve these outcomes, especially if a bifurcated stent-graft is implanted.

Keywords: Abdominal aortic aneurysm. Ruptured aneurysm. EVAR. Open surgical repair. Mortality. 


\section{INTRODUCCIÓN}

El aneurisma de aorta abdominal se define como una dilatación de la aorta a dicho nivel a un diámetro de $30 \mathrm{~mm}$ o superior (1). Si dicha alteración no se corrige de forma programada, el progresivo aumento de tamaño del aneurisma puede llevar a su rotura, entidad clínica que se asocia a elevadas cifras de morbimortalidad (50-70 \%) (2).

La introducción de técnicas endovasculares (EVAR) en la reparación de los aneurismas de aorta abdominal rotos surgió como alternativa a la cirugía abierta convencional y hoy se considera como primera opción en casos con anatomía favorable con un nivel de evidencia 1b (1).

Si bien el EVAR ha demostrado su superioridad respecto a la cirugía abierta en términos de morbimortalidad perioperatoria con relación a la cirugía abierta en la cirugía programada (3), estos hallazgos no han sido sustentados por ensayos clínicos aleatorizados en el AAAr (4).

No obstante, estudios observacionales han demostrado tasas de mortalidad y complicaciones que, si bien no son estadísticamente significativas, muestran una tendencia que apoya el uso del EVAR como primera opción terapéutica en el $\operatorname{AAAr}(2,5,6)$. Además, sí que podemos encontrar en la literatura estudios observacionales y series de casos en los que sí se hallaron diferencias estadísticamente significativas en favor del abordaje mínimamente invasivo (7$11,14)$, por lo que como se ha comentado previamente, las guías de práctica clínica actuales recomiendan el EVAR como terapia de primera línea siempre que este sea factible (1).

Por tanto, y dada la gran disparidad de datos respecto al tratamiento de esta patología y sus resultados, el objetivo de nuestro estudio es analizar las técnicas de tratamiento del aneurisma infrarrenal roto en nuestro centro, comparando cirugía abierta (CA) y endovascular (EVAR) en términos de morbimortalidad perioperatoria y posoperatoria, así como la supervivencia en el seguimiento.

\section{MATERIAL Y MÉTODOS}

Se realizó un estudio retrospectivo de pacientes con aneurisma de aorta abdominal roto intervenidos de manera consecutiva entre enero de 2006 y diciembre de 2017 en el Hospital Clínico San Carlos, centro de referencia del 
área 7 de Madrid, con un volumen de población de 370.501 personas, según se recoge en la memoria del hospital del año 2017.

Destacar que, en nuestro centro, ante la sospecha de esta patología mediante la clínica y ecografía abdominal realizada a pie de cama en el Servicio de Urgencias, se realiza inicialmente una monitorización estrecha del paciente, se extrae analítica completa y gasometría, se canalizan 2 vías periféricas de gran calibre y se solicitan pruebas cruzadas urgentes a la vez que se avisa con carácter de emergencia a nuestro servicio para la valoración del caso. Si el paciente se considera operable en base a su edad y comorbilidades se realiza una angioTC si la situación hemodinámica lo permite. Tras la realización del angioTC se analizan las imágenes en la estación de trabajo para valorar criterios morfológicos y decidir qué tipo de intervención llevar a cabo. Durante todo el manejo perioperatorio se lleva a cabo una estrategia de hipotensión permisiva (TAS $70-90 \mathrm{mmHg}$ ). Todos los procedimientos realizados mediante cirugía abierta son llevados a cabo bajo anestesia general. Por otra parte, los pacientes en los que se decida EVAR, inicialmente son intervenidos bajo anestesia local, si bien se realiza conversión a anestesia general en el caso de que sean poco colaboradores o presenten un empeoramiento clínico o hemodinámico brusco al inicio de la intervención.

Fueron incluidos todos aquellos pacientes con aneurisma de aorta abdominal roto con cuello infrarrenal de al menos $10 \mathrm{~mm}$ de longitud objetivado mediante angioTC. Se excluyeron aquellos pacientes con aneurisma de aorta abdominal micótico, yuxtarrenal, toracoabdominal e iliaco aislado, bien por diferencias basales en cuanto a pronóstico o porque no reunieran las características anatómicas necesarias que permitieran o requirieran la implantación de un EVAR convencional.

La muestra fue dividida en 2 cohortes en función de si los pacientes fueron intervenidos mediante cirugía abierta o EVAR. Dado que todos los pacientes reunían criterios anatómicos y morfológicos que permitían la implantación de una endoprótesis, la decisión de realizar una técnica quirúrgica u otra se basó en la preferencia del cirujano principal en base a su experiencia en ambas técnicas, así como de la disponibilidad en el centro de la endoprótesis adecuada para el caso. La decisión no se basó en la situación hemodinámica del paciente previa a la intervención. 
Todos aquellos pacientes en los que no fue posible la realización de una angioTC preoperatorio por su situación hemodinámica fueron intervenidos mediante cirugía abierta, si bien no fueron incluidos en el estudio por no presentar una prueba de imagen preoperatoria en la que poder objetivar si se podría implantar un EVAR convencional. En el grupo endovascular se incluyeron pacientes tratados tanto con endoprótesis bifurcada como con endoprótesis aortouniiliaca más bypass femorofemoral cruzado. Los procedimientos endovasculares fueron llevados a cabo en su totalidad en un quirófano convencional equipado con una mesa de operaciones radiotransparente, mediante el uso de un arco en C móvil y un inyector de contraste de alta presión.

Inicialmente se realizó un estudio descriptivo por grupos en cuanto a características demográficas y comorbilidades. Posteriormente se compararon características del aneurisma y de la cirugía tales como el diámetro del aneurisma, la presencia de aneurisma iliaco asociado, el tipo de anestesia, las necesidades transfusionales y la presencia de inestabilidad hemodinámica en el momento de la intervención, definida como necesidad de intubación orotraqueal preoperatoria por pérdida de consciencia o hipotensión y taquicardia que precisaran de fármacos vasoactivos.

El objetivo primario del estudio fue evaluar si existieron diferencias en cuanto a mortalidad intraoperatoria y hospitalaria. Los objetivos secundarios comprendieron el análisis de las complicaciones mayores, la tasa de reintervención precoz, la supervivencia durante el seguimiento, así como la evolución histórica de la técnica quirúrgica.

Se empleó el paquete estadístico SPSS Statistics 22.0. Para el análisis estadístico de las comorbilidades, variables perioperatorias y resultados postoperatorios se emplearon la t de Student, chi-cuadrado, test de Breslow y regresión de Cox. Se realizó asimismo una curva de supervivencia de KaplanMeier, aplicando un log-rank para valorar su significación estadística.

\section{RESULTADOS}

Entre enero de 2006 y diciembre de 2017, 95 pacientes fueron intervenidos en nuestro centro por aneurisma roto, de los cuales a 82 fue posible realizarles angioTC preoperatorio. Fueron excluidos 23 pacientes por aneurismas con cuello menor de $10 \mathrm{~mm}, 2$ por aneurisma toracoabdominal, 2 por aneurisma 
iliaco aislado y 1 por aneurisma micótico. Por tanto, finalmente fueron incluidos 54 pacientes, 26 intervenidos mediante cirugía abierta $(48,1 \%)$ y 28 mediante $\operatorname{EVAR}(51,9 \%)$.

Durante este periodo se ha producido un aumento paulatino del número de casos intervenidos de forma endovascular en detrimento de la cirugía abierta. Dentro del grupo EVAR se observa progresivamente un mayor porcentaje de pacientes tratados mediante endoprótesis bifurcada (Fig. 1).

En cuanto a características demográficas y comorbilidades, la edad media de los pacientes intervenidos fue de 70,7 \pm 7,8 años en el grupo CA y de 72,5 \pm 9,5 años en el grupo $\operatorname{EVAR}(p=0,45)$. La mayoría de los pacientes tratados fueron varones, lo cual representa un $96,2 \%$ en el grupo CA y un $92,9 \%$ en el grupo EVAR. Estos datos, así como los factores de riesgo cardiovascular y principales comorbilidades expresados en términos de frecuencia absoluta y relativa, aparecen reflejados en la tabla $I$. No se hallaron diferencias estadísticamente significativas en estas variables, a excepción de la dislipemia, presente en un $26,9 \%$ de pacientes del grupo CA (7/26) y en un $67,9 \%$ de pacientes del grupo EVAR (19/28), con una $p=0,003$.

Respecto a las características del aneurisma, los tratados en el grupo CA eran más grandes que los del grupo EVAR $(88,1 \pm 17,9 \mathrm{~mm}$ vs. $72,5 \pm 16,1 \mathrm{~mm}, \mathrm{p}=$ 0,02 ). Asimismo, en el grupo CA hubo un mayor porcentaje de aneurisma asociado a nivel de la arteria iliaca común, si bien esta diferencia no fue estadísticamente significativa (34,6 \% vs. 17,8\%, $p=0,07)$.

En relación a la cirugía, todos los procedimientos del grupo CA se realizaron con anestesia general, mientras que en el grupo EVAR un $50 \%$ se llevaron a cabo mediante anestesia local $(p=0,001)$. Un $65,4 \%$ de los pacientes del grupo CA (17/26) presentaban inestabilidad hemodinámica en el momento de la intervención por un $60,7 \%(17 / 28)$ del grupo EVAR $(p=0,72)$. Las necesidades transfusionales intraoperatorias medianas, medidas en concentrados de hematíes $(\mathrm{CH})$, fueron de 11 para el grupo CA y 4 para el grupo EVAR ( $p=0,001)$. El éxito técnico en el grupo EVAR fue del $100 \%$, y no hubo ningún caso de conversión a cirugía abierta. En el grupo CA hubo 4 éxitus intraoperatorios, todos ellos tras el clampaje aórtico proximal, antes de la realización de la anastomosis proximal del bypass (Tabla II). Respecto a los 22 pacientes restantes de dicho grupo, a 13 se les realizó un bypass aortobifemoral, a 8 un bypass aortoaórtico y a 1 un bypass aortobiiliaco. 
La mortalidad hospitalaria fue de $12 / 26$ (46,2 \%) en el grupo CA frente a 8/28 $(28,6 \%)$ en el grupo EVAR, lo cual no es un resultado estadísticamente significativo $(p=0,18)$.

La tasa de reintervención precoz fue de 4/22 (18,2\%) en el grupo CA y 7/28 $(25 \%)$ en el grupo EVAR $(p=0,56)$. En el grupo CA dichas reintervenciones se debieron en 3 casos a colitis isquémica que requirieron resección intestinal y en otro de ellos a un sangrado procedente de la anastomosis distal de un bypass aortoaórtico. En el grupo EVAR hubo 1 caso de laparotomía secundaria a síndrome compartimental, mientras que los 6 restantes se debieron a complicaciones secundarias a la cirugía. Estas fueron una trombosis del EVAR, una trombosis del bypass femorofemoral, una trombosis distal de MMII, una fuga tipo la, una infección del bypass femorofemoral en el vigésimo noveno día posoperatorio, realizándose retirada del mismo y un nuevo bypass axilofemoral izquierdo, y una rotura de pseudoaneurisma femoral en el decimocuarto día tras la intervención, rehaciéndose la anastomosis a dicho nivel. Destacar que todas las reintervenciones llevadas a cabo en dicho grupo fueron en el subgrupo de endoprótesis aortouniiliaca.

La tasa de complicaciones mayores posoperatorias fue del $54,5 \%$ en el grupo CA y del $64,3 \%$ en el grupo EVAR $(p=0,48)$. Dentro de ellas las dos más frecuentes fueron el síndrome de distrés respiratorio agudo (SDRA) y el fracaso renal agudo (FRA). El primero afectó a un 45,4 \% (10/22) y un 39,3\% (11/28) de los pacientes de los grupos CA y EVAR respectivamente, requiriendo los pacientes de una intubación prolongada. El segundo estuvo presente en un $63,6 \%(14 / 22)$ del grupo CA y 53,6 \% (15/28) del grupo EVAR, siendo necesario en 3 casos de cada grupo terapia de reemplazo renal transitoria. Destacar asimismo que hubo 4 casos de colitis isquémica en el grupo CA $(18,2 \%)$ y ninguno en el EVAR, con una $p=0,07$. El resto de complicaciones mayores posquirúrgicas aparecen reflejadas en la tabla III.

Por último, se realizó un análisis de supervivencia, que fue en el seguimiento a 2 años del $51,7 \%$ en el grupo CA y del 65,2 \% en el grupo EVAR, lo cual no es un resultado estadísticamente significativo $(p=0,28)$ (Fig. 2 ).

\section{DISCUSIÓN}

El aneurisma de aorta abdominal roto representa una patología con una alta morbimortalidad asociada. En la literatura se pueden encontrar numerosas 
publicaciones comparando las dos estrategias quirúrgicas existentes, con resultados y conclusiones variados.

Esta disparidad puede deberse a varios motivos. En primer lugar, dado que se trata de una patología con una baja incidencia, los tamaños muestrales de los estudios pueden no ser lo suficientemente grandes como para hallar diferencias significativas. En segundo lugar, pueden existir limitaciones desde el punto de vista de la recogida de datos en cuanto a la situación hemodinámica de los pacientes a su llegada a los servicios de Urgencias y la adecuada exposición de las complicaciones posoperatorias en el historial médico. Por último, al tratarse de patología en la que la técnica quirúrgica elegida depende en gran medida de la situación clínica del paciente, no resulta sencillo realizar un ensayo clínico aleatorizado por los aspectos éticos que acarrearía.

En cuanto al objetivo principal del estudio, que es la mortalidad hospitalaria, existe una gran controversia en la literatura. En el metaanálisis de van Beek y cols. (4) del año 2014, en el que se incluyeron tanto ensayos clínicos aleatorizados como estudios observacionales, el análisis de la mortalidad intrahospitalaria por subgrupos demostró resultados dispares. Mientras que en el caso de los ensayos clínicos aleatorizados no se hallaron diferencias significativas, el análisis de los estudios observacionales mostró resultados favorables hacia el EVAR, si bien es cierto que este segundo grupo presentó una mayor heterogeneidad y un mayor riesgo de sesgos, además de pequeños tamaños muestrales.

Sin embargo, en el metaanálisis de Quin y cols., publicado en el mismo año y en el que se incluyeron también ambos tipos de estudios, sí se demostraron diferencias significativas a favor del EVAR en cuanto a mortalidad en los primeros 30 días tras la intervención (14). En nuestro estudio la mortalidad hospitalaria fue del $46,2 \%$ en el grupo CA frente al $28,6 \%$ en el grupo EVAR, no siendo esta diferencia significativa. Estos resultados indican que ambos procedimientos serían válidos y equiparables en el tratamiento del AAAr en términos de mortalidad perioperatoria, si bien se muestra una cierta tendencia favorable hacia la terapia endovascular. A pesar de nuestro pequeño tamaño muestral, podemos encontrar algunos factores que podrían explicar esta tendencia. 
En primer lugar, en nuestra serie, un $50 \%$ de los procedimientos del grupo EVAR se realizaron bajo anestesia local. Esta estrategia se ha asociado a una baja tasa de mortalidad posoperatoria (15), probablemente debido a dos razones. Inicialmente, al evitar la inducción anestésica no se produce la relajación de la musculatura abdominal y con ello disminuye el riesgo de progresión de la rotura aórtica. Posteriormente, el empleo de anestesia general inhibe el sistema nervioso simpático, haciendo aún más difícil si cabe el control tensional en pacientes hemodinámicamente muy lábiles.

En segundo lugar, las necesidades transfusionales intraoperatorias fueron mayores en el grupo CA (11 vs. 4), siendo esta diferencia significativa. Esto podría deberse a unas mayores pérdidas sanguíneas en el caso de la cirugía abierta debido a la apertura del retroperitoneo (5), con un mayor número de pacientes en este grupo que requería transfusión de hemoderivados (8), lo cual se asocia a una mayor morbilidad en el posoperatorio según publicaron en 2017 Kordzadeh y cols. (16), definiendo morbilidad en dicho estudio como complicaciones mayores que requiriesen una intervención quirúrgica, endoscópica o radiológica, o bien fallos orgánicos que amenazasen la vida del paciente.

La inestabilidad hemodinámica ha demostrado ser factor independiente de mortalidad (6) así como factor de confusión, ya que en pacientes en esta situación no siempre es posible realizar una prueba de imagen preoperatoria. De hecho, en el metaanálisis de Luebke y cols. del año 2015, en el que inicialmente la terapia EVAR mostró una menor mortalidad a 30 días, no se hallaron diferencias significativas posteriormente entre EVAR y CA tras realizar un ajuste en base a la situación hemodinámica preoperatoria (17). Esto no supone un sesgo en nuestro estudio, ya que la inestabilidad hemodinámica estaba presente por igual en ambos grupos (65,4 \% CA vs. 60,7 \% EVAR), y todos los pacientes incluidos presentaban angioTC preoperatorio.

Otro aspecto a destacar de los resultados es el auge del tratamiento endovascular con el paso de los años, y dentro de este, de las prótesis bifurcadas. Marin y Veith describieron por primera vez en 1995 el uso de una endoprótesis aortouniiliaca, oclusor en iliaca común contralateral y bypass femorofemoral cruzado para el tratamiento de la patología aneurismática aortoiliaca (12). 
Sin embargo, el uso de estos dispositivos ha demostrado una mayor tasa de mortalidad, reintervención y complicaciones a nivel de la herida quirúrgica en comparación con los dispositivos bifurcados (13). Estos datos se encuentran en consonancia con nuestros resultados. Si bien no hemos analizado la mortalidad por el pequeño tamaño muestral, los 7 casos de reintervenciones en el grupo EVAR se llevaron a cabo en el subgrupo de dispositivos aortouniiliacos, probablemente debido a una mayor manipulación quirúrgica a nivel de las bifurcaciones femorales, y a la tunelización subcutánea de materiales protésicos.

En cuanto a las complicaciones posoperatorias, destacar el caso de la colitis isquémica. Si bien la diferencia entre ambos grupos no resultó estadísticamente significativa $(p=0,07$ ), podría haber dos factores que influyeran en una mayor tasa de esta complicación en los pacientes intervenidos mediante cirugía abierta. Por un lado, debido a que todos los procedimientos en este grupo se realizaron bajo anestesia general frente a un $50 \%$ en el caso del grupo EVAR, esto podría influir en que los pacientes presentaran una mayor labilidad hemodinámica durante la cirugía, y por tanto un mayor riesgo de desarrollar isquemia intestinal por bajo gasto. Por otro lado, la mayor pérdida sanguínea en este grupo con necesidades transfusionales mayores podría contribuir a la isquemia del colon secundaria a hipovolemia. No obstante, no identificamos una inestabilidad hemodinámica significativamente mayor en el grupo de pacientes con cirugía abierta, por lo que este será un aspecto a evaluar con mayor detalle en futuros estudios.

En relación a la supervivencia a 2 años, nuestros resultados no demostraron diferencias significativas $(51,7 \%$ CA vs. 65,2 \% EVAR, $p=0,28)$. Este punto también representa una importante controversia. Mientras que el estudio IMPROVE demostró un beneficio en favor del EVAR 3 años tras la intervención en términos de supervivencia y calidad de vida (18), podemos encontrar en la literatura estudios en los cuales ambas técnicas quirúrgicas no presentan diferencias en cuanto a supervivencia a partir del periodo perioperatorio $(19,20)$. Reite y cols. describieron que la supervivencia en pacientes tratados mediante cirugía abierta sería equiparable a la de la población general para aquellos pacientes que sobreviven los primeros 30 días tras la intervención (21). 
Por último, como principales limitaciones de nuestro estudio destacar inicialmente el carácter retrospectivo del mismo, con la consiguiente pérdida de información a la hora de la recogida de datos. Asimismo, destacar el pequeño tamaño muestral previamente mencionado, debido en gran parte a la exclusión de aneurismas rotos yuxtarrenales para hacer ambos grupos comparables, que quizás limite la posibilidad de hallar diferencias significativas.

\section{CONCLUSIÓN}

El tratamiento del aneurisma de aorta infrarrenal roto continúa siendo un importante desafío para el cirujano vascular, ya que se trata de una patología que sigue asociando unas elevadas tasas de morbimortalidad con independencia de la técnica quirúrgica empleada. Nuestros resultados muestran que ambas alternativas quirúrgicas serían comparables, si bien parecen mostrar una tendencia favorable hacia el EVAR respecto a la cirugía abierta. Dentro del tratamiento endovascular, las endoprótesis bifurcadas parecen aportar ciertas ventajas respecto a los dispositivos aortouniiliacos.

\section{BIBLIOGRAFÍA}

1. Wanhainen A, Verzini F, Van Herzeele I, Allaire E, Bown M, Cohnert T, et al. European Society for Vascular Surgery (ESVS) 2019 Clinical Practice Guidelines on the Management of Abdominal Aorto-iliac Artery Aneurysms. Eur J Vasc Endovasc Surg 2019;57(1):8-93. DOI: 10.1016/j.ejvs.2018.09.020

2. Badger S, Bedenis R, Blair PH, Ellis P, Kee F, Harkin DW. Endovascular treatment for ruptured abdominal aortic aneurysm. Cochrane Database Syst Rev 2014;(7):CD005261. DOI: 10.1002/14651858.CD005261.pub4

3. Propper BW, Abularrage CJ. Long-term safety and efficacy of endovascular abdominal aortic aneurysm repair. Vasc Health Risk Manag 2013;9:135-41. DOI: 10.2147/VHRM.S32250

4. van Beek SC, Conijn AP, Koelemay MJ, Balm R. Editor's Choice Endovascular aneurysm repair versus open repair for patients with a ruptured abdominal aortic aneurysm: a systematic review and meta- 
analysis of short-term survival. Eur J Vasc Endovasc Surg 2014;47(6):593602. DOI: 10.1016/j.ejvs.2014.03.003

5. Reimerink JJ, Hoornweg LL, Vahl AC, Wisselink W, van den Broek TA, Legemate DA, et al. Endovascular Repair Versus Open Repair of Ruptured Abdominal Aortic Aneurysms. A Multicenter Randomized Controlled Trial. Ann Surg 2013;258(2):248-56. DOI: 10.1097/SLA.0b013e31828d4b76

6. Del Canto Peruyera P, Alvarez Salgado A, Calvín Alvarez P, Botas Velasco $M$, Vallina-Victorero Vázquez MJ, et al. Tratamiento del aneurisma de aorta abdominal roto: ¿EVAR o cirugía abierta? Angiología 2014;66(6):300-4. DOI: 10.1016/j.angio.2014.05.006

7. Portelli Tremont JN, Cha A, Dombrovs.kiy VY, Rahimi SA. Endovascular Repair for Ruptured Abdominal Aortic Aneurysms has Improved Outcomes Compared to Open Surgical Repair. Vasc Endovascular Surg 2016;50(3):147-55. DOI: 10.1177/1538574416637442

8. Tan TW, Eslami M, Rybin D, Doros G, Zhang WW, Farber A. Outcomes of endovascular and open surgical repair of ruptured abdominal aortic aneurysms in elderly patients. J Vasc Surg 2017;66(1):64-70. DOI: 10.1016/j.jvs.2016.10.119

9. Gupta AK, Dakour Aridi H, Locham S, Nejim B, Veith FJ, Malas MB. Realworld evidence of superiority of endovascular repair in treating ruptured abdominal aortic aneurysm. J Vasc surg 2018;68(1):74-81. DOI: 10.1016/j.jvs.2017.11.065

10. Giles KA, Pomposelli FB, Hamdan AD, Wyers MC, Schermerhorn ML. Comparison of Open and Endovascular Repair of Ruptured Abdominal Aortic Aneurysms From the ACS-NSQIP 2005-07. J Endovasc Ther 2009;16(3):365-72. DOI: 10.1583/09-2735.1

11. Nedeau AE, Pomposelli FB, Hamdan AD, Wyers MC, Hsu R, Sachs T, et al. Endovascular vs. open repair for ruptured abdominal aortic aneurysm. J Vasc Surg 2012;56(1):15-20. DOI: 10.1016/j.jvs.2011.12.067

12. Marin ML, Veith FJ, Cynamon J, Sanchez LA, Lyon RT, Levine BA, et al. Initial experience with transluminally placed endovascular grafts for the treatment of complex vascular lesions. Ann Surg 1995;222(4):449-69. DOI: 10.1097/00000658-199522240-00004

13. Gupta PK, Kempe K, Brahmbhatt R, Gupta H, Montes J, Forse RA, et al. Outcomes After Use of Aortouniiliac Endoprosthesis Versus Modular or 
Unibody Bifurcated Endoprostheses for Endovascular Repair of Ruptured Abdominal Aortic Aneurysms. Vasc Endovascular Surg 2017;51(6):357-62. DOI: $10.1177 / 1538574417703562$

14. Quin C, Chen L, Xiao YB. Emergent Endovascular vs. Open Surgery Repair for Ruptured Abdominal Aortic Aneurysms: A Meta-Analysis. PLoS One 2014;9(1):e87465. DOI: 10.1371/journal.pone.0087465

15. Lachat ML, Pfammatter T, Witzke HJ, Bettex D, Kuenzli A, Wolfensberger $U$, et al. Endovascular Repair with Bifurcated Stent-Grafts under Local Anaesthesia to Improve Outcome of Ruptured Aortoiliac Aneurysms. Eur J Vasc Endovasc Surg 2002;23(6):528-36. DOI: 10.1053/ejvs.2002.1622

16. Kordzadeh A, Askari A, Parsa AD, Browne T, Panayiotopoulos YP. The Clinical Implication of Blood Product Transfusion on Morbidity and Mortality of Ruptured Abdominal Aortic Aneurysm. Clin Appl Thromb Hemost 2017;23(6):601-6. DOI: 10.1177/1076029615624548

17. Luebke T, Brunkwall J. Risk-Adjusted Meta-analysis of 30-Day Mortality of Endovascular Versus Open Repair for Ruptured Abdominal Aortic Aneurysms. Ann Vasc Surg 2015;29(4):845-63. DOI: 10.1016/j.avsg.2014.12.014

18. Ulug $P$, Hinchliffe RJ, Sweetling MJ, Gomes M, Thompson MT, Thompson SG, et al. Strategy of endovascular versus open repair for patients with clinical diagnosis of ruptured abdominal aortic aneurysm: the IMPROVE RCT. Health Technol Assess 2018;22(31):1-122. DOI: 10.3310/hta22310

19. Lundgren $\mathrm{F}$, Troeng $\mathrm{T}$. Treatment choice and survival after ruptured abdominal aortic aneurysm: A population-based study. J Vasc Surg 2020;72(2):508-17. DOI: 10.1016/j.jvs.2019.11.060

20. Salata K, Hussain MA, de Mestral C, Greco E, Awartani H, Aljabri BA, et al. Population-based long-term outcomes of open versus endovascular aortic repair of ruptured abdominal aortic aneurysms. J Vasc Surg 2020;71(6):1867-78. DOI: 10.1016/j.jvs.2019.06.212

21. Reite A, Soreide K, Kvaloy JT, Vetrhus M. Long-Term Outcomes After Open Repair for Ruptured Abdominal Aortic Aneurysm. World J Surg 2020;44(6):2020-7. DOI: 10.1007/s00268-020-05457-7 
Tabla I. Factores demográficos y de riesgo cardiovascular y comorbilidades

\begin{tabular}{|l|l|l|l|}
\hline & CA $(n=26)$ & EVAR $(n=28)$ & $p$ \\
\hline Varón & $25(96,2 \%)$ & $26(92,9 \%)$ & 0,59 \\
\hline Edad & $70,7 \pm 7,8$ & $72,5 \pm 9,5$ & 0,45 \\
\hline HTA & $18(69,2 \%)$ & $21(75 \%)$ & 0,64 \\
\hline Tabaco & $9(34,6 \%)$ & $5(17,9 \%)$ & 0,33 \\
\hline Dislipemia & $7(26,9 \%)$ & $19(67,9 \%)$ & 0,003 \\
\hline DM & $0(0 \%)$ & $3(10,7 \%)$ & 0,23 \\
\hline Cardiopatía & $4(15,4 \%)$ & $6(21,4 \%)$ & 0,57 \\
isquémica & & & \\
\hline PCV & $1(3,8 \%)$ & $3(10,7 \%)$ & 0,34 \\
\hline IRC & $11(42,3 \%)$ & $15(53,6 \%)$ & 0,80 \\
\hline EPOC & $4(15,4 \%)$ & $7(25 \%)$ & 0,38 \\
\hline FA & $5(19,2 \%)$ & $4(14,3 \%)$ & 0,63 \\
\hline EAP & $2(7,7 \%)$ & $3(10,7 \%)$ & 0,65 \\
\hline EO
\end{tabular}

No se objetivaron diferencias estadísticamente significativas entre ambos grupos, a excepción de la dislipemia. HTA: hipertensión arterial; DM: diabetes mellitus; PCV: patología cerebrovascular; IRC: insuficiencia renal crónica; EPOC: enfermedad pulmonar obstructiva crónica; FA: fibrilación auricular; EAP: enfermedad arterial periférica. 
Tabla II. Características del aneurisma y variables perioperatorias e intraoperatorias

\begin{tabular}{|l|l|l|l|l|}
\hline \multicolumn{2}{|l|}{} & CA $(\mathrm{n}=26)$ & $\begin{array}{l}\text { EVAR }(\mathrm{n}=\mathrm{p} \\
28)\end{array}$ & \\
\hline Tipo de anestesia & Local & $0(0 \%)$ & $14(50 \%)$ & 0,001 \\
\cline { 2 - 5 } & General & $26(100 \%)$ & $14(50 \%)$ & \\
\hline Diámetro del aneurisma (mm) & $88,1 \pm 17,9$ & $72,5 \pm 16,1$ & 0,02 \\
\hline Aneurisma iliaco asociado & $9(34,6 \%)$ & $5(17,8 \%)$ & 0,07 \\
\hline Necesidades & Mediana & 11 & 4 & 0,001 \\
transfusiones (CH) & Rango intercuartil & 12 & 6 & \\
\hline Inestabilidad hemodinámica & $17(65,4 \%)$ & $17(60,7 \%)$ & 0,72 \\
\hline Éxitus intraoperatorio & $4(15,4 \%)$ & $0(0 \%)$ & 0,03 \\
\hline Mortalidad hospitalaria & $12(46,2 \%)$ & $8(28,6 \%)$ & 0,18 \\
\hline
\end{tabular}

$\mathrm{CH}$ : concentrado de hematíes.

Tabla III. Tasa de reintervención precoz y complicaciones mayores con subtipos 


\begin{tabular}{|c|c|c|c|c|}
\hline & \multicolumn{3}{|c|}{ CA $(n=22)$ EVAR $\quad(n=p$} \\
\hline \multicolumn{2}{|c|}{ Reintervención precoz } & $4(18,2 \%)$ & $7(25 \%)$ & 0,56 \\
\hline \multicolumn{2}{|c|}{ Complicaciones mayores } & $12(54,5 \%)$ & $18(64,3 \%)$ & 0,48 \\
\hline \multirow[t]{2}{*}{ Cardiológicas } & Shock cardiogénico & $9(40,9 \%)$ & $7(25 \%)$ & 0,21 \\
\hline & IAM & $1(4,5 \%)$ & $0(0 \%)$ & 0,33 \\
\hline \multirow[t]{2}{*}{ Pulmonares } & SDRA & $10(45,4 \%)$ & $11(39,3 \%)$ & 0,57 \\
\hline & Neumonía & $5(22,7 \%)$ & $5(17,9 \%)$ & 0,72 \\
\hline \multirow[t]{2}{*}{ Renales } & FRA & $11(50 \%)$ & $12(42,9 \%)$ & 0,69 \\
\hline & TSR & $3(13,6 \%)$ & $3(10,7 \%)$ & 0,81 \\
\hline \multirow[t]{2}{*}{ Digestivas } & Colitis isquémica & $4(18,2 \%)$ & $0(0 \%)$ & 0,07 \\
\hline & HDA & $2(9,1 \%)$ & $1(3,6 \%)$ & 0,43 \\
\hline \multicolumn{2}{|l|}{ Neurológicas } & $4(18,2 \%)$ & $4(14,3 \%)$ & 0,67 \\
\hline \multirow{2}{*}{\multicolumn{2}{|c|}{$\begin{array}{l}\text { Infección HQ } \\
\text { TFP }\end{array}$}} & $1(4,5 \%)$ & $5(17,9 \%)$ & 0,06 \\
\hline & & $0(0 \%)$ & $2(7,1 \%)$ & 0,25 \\
\hline
\end{tabular}

Todas las reintervenciones en el grupo EVAR se realizaron en el subgrupo de endoprótesis aortomonoiliaca. IAM: infarto agudo de miocardio; SDRA: síndrome de distrés respiratorio agudo; FRA: fracaso renal agudo; TSR: tratamiento sustitutivo renal; HDA: hemorragia digestiva alta; HQ: herida quirúrgica; TEP: tromboembolismo pulmonar. 


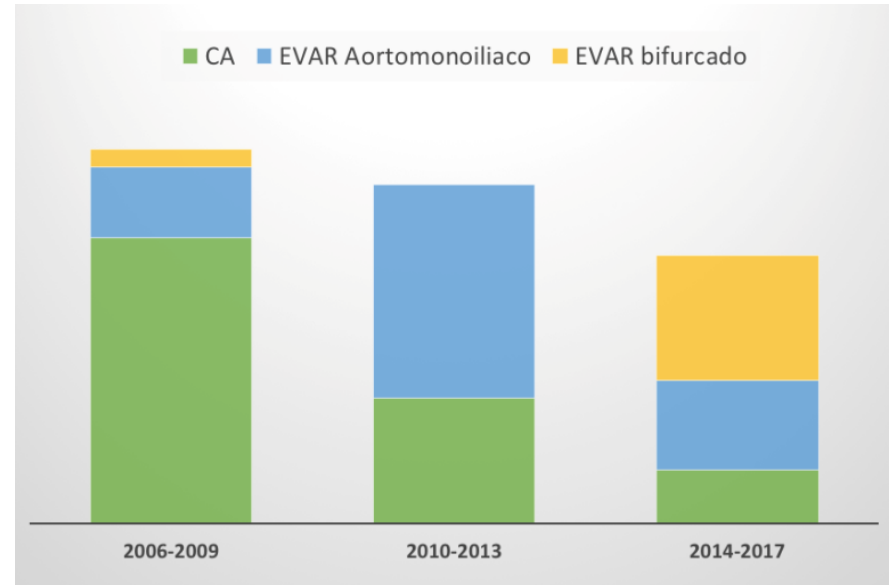

Figura 1. Evolución de la técnica quirúrgica a lo largo del periodo analizado.

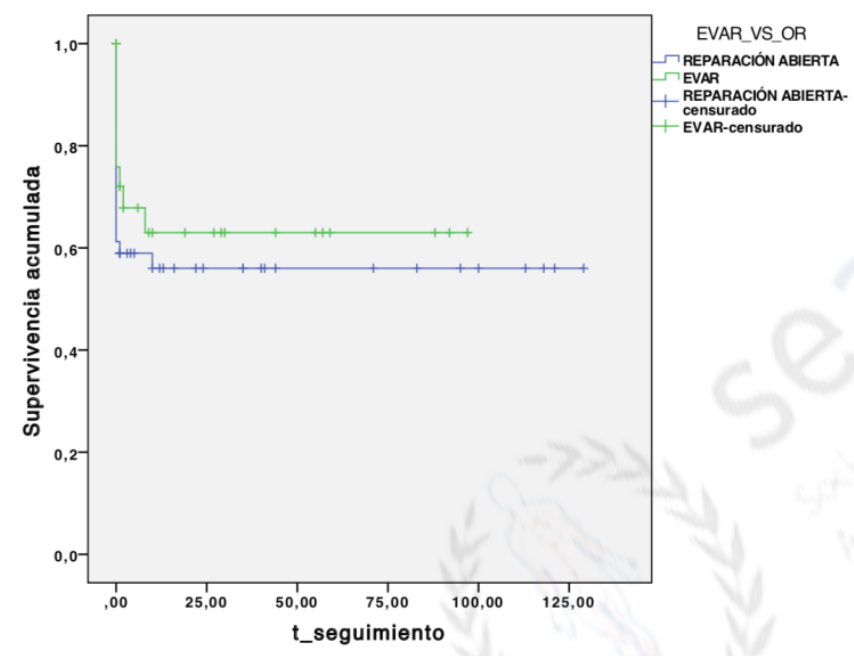

Figura 2. Análisis de supervivencia comparando ambos grupos. Se objetiva una mayor supervivencia en el grupo EVAR durante el seguimiento, no estadísticamente significativa ( $p=0.28)$, dependiente de una mayor mortalidad del grupo CA en el posoperatorio inmediato y primeros meses tras la intervención. 